\title{
Epidemiology and prevention strategies of SARS-CoV-2 infection in pediatric hematology and oncology centers in Poland
}

\begin{abstract}
Introduction: Epidemiological analysis of severe acute respiratory syndrome coronavirus-2 (SARS-CoV-2) infections in pediatric hematology and oncology ( $\mathrm{PHO}$ ) and hematopoietic cell transplant (HCT) centers in a Polish nationwide study, as well as analysis of the preventive strategies in these centers. Methods: All of the $18 \mathrm{PHO} / \mathrm{HCT}$ centers participated in eight surveys and questionnaires conducted over the first 5 months of the SARS-CoV-2/ coronavirus disease 2019 (COVID-19) pandemic in Poland. Epidemiological data were collected at eight regular time points, and the strategy of preventive management was done once after 4 months of the pandemic. Results: During this analyzed period, eight patients were positive for SARS-CoV-2. The estimated incidence of SARS-CoV-2 positivity in Polish PHO/HCT centers was $0.5 \%$. After exclusion of HCT patients (with one patient being infected), the estimated incidence of SARS CoV-2 positivity was between 0.5 and $0.6 \%$. In all but one case, the course of COVID-19 was asymptomatic or mild, and it was moderate in one case. None of them developed SARS or respiratory insufficiency, none of them required treatment in the intensive care unit (ICU), and no patient died due to SARS-CoV-2 infection. As of July 1, parents staying in the hospital together with their children were regularly tested for the virus in 13 centers. Asymptomatic healthcare personnel were regularly tested for the virus in seven centers. Conclusions: The estimated incidence of SARS-CoV-2 infection among PHO/HCT patients is lower than in Western Europe; however, these patients cannot be regarded as a low-risk group. The low COVID-19 incidence should be interpreted as a result of strictly and continuously implemented detailed preventive measures in the PHO/HCT wards and in hospitals.
\end{abstract}

(c) 2020 Polish Society of Hematology and Transfusion Medicine, Insitute of Hematology and Transfusion Medicine. Published by Sciendo. All rights reserved.

Keywords:

pediatric oncology, children, SARS-CoV-2, COVID-19, epidemiology, outcome

\section{Introduction}

Infections with a new coronavirus severe acute respiratory syndrome coronavirus-2 (SARS-CoV-2) first started in the Chinese district of Wuhan, and then spread rapidly throughout the entire world. The World Health Organization (WHO) announced the pandemic on March 11, and called the disease as coronavirus disease 2019 (COVID-19). Several countries noted very high rates of deaths caused by severe acute respiratory syndrome (SARS) [1]. The Polish Ministry of Health announced the epidemic crisis situation on March 14, and epidemic on March 23, 2020. This pandemic caused an unprecedented situation and stress on the healthcare system in all countries. Patient groups at highest risk for severe disease included age, comorbidities, and predisposing conditions such as immune suppression [2, 3]. Thus, all patients undergoing anticancer and/or immunosuppressive therapy faced the risk of infection $[4,5]$ and potential severe disease course and complications [6, 7]. The objective of this study is epidemiological analysis of the incidence of infections with SARS-CoV-2 in pediatric hematology and oncology $(\mathrm{PHO})$ centers in a Polish nationwide study, as well as analysis of the preventive strategies in these centers.

\section{Methods}

\section{Study design}

The study consisted of two parts: (1) epidemiological data at eight time points and (2) strategy of prevention and management (as of July 1). Epidemiological data were regularly collected in eight time points between March 1 and August 1 , initially on a 2-week basis, then from June 1, on monthly reports. The following data were collected: the number of patients at risk (on intensive chemotherapy, on immunosuppression, requiring visits in hospital); number of patients tested for SARS-CoV-2; number of patients positive for SARS-CoV-2; number of patients with SARS-CoV-2 requiring therapy in the intensive care unit (ICU); and number of patients who died due to SARS-CoV-2. To
Article history:

Received: 15.08.2020 Accepted: 21.08 .2020

Jan Styczyński ${ }^{1, *}$ Walentyna Balwierz ${ }^{2}$, Jacek Wachowiak Krzysztof Kałwak ${ }^{4}$ Bernarda Kazanowska ${ }^{4}$, Wojciech Młynarski ${ }^{5}$, Bożenna Dembowska-Bagińska ${ }^{6}$, Michał Matysiak? Maryna Krawczuk-Rybak ${ }^{8}$, Elżbieta Adamkiewicz-Drożyńska9 ${ }^{9}$ Jerzy Kowalczyk ${ }^{10}$ Anna Raciborska ${ }^{11}$ Tomasz Urasiński'2, Jarosław Peregud-Pogorzelski ${ }^{13}$, Radosław Chaber $^{14}$, Wanda Badowska ${ }^{15}$ Grażyna Karolczyk ${ }^{16}$ Agnieszka Mizia-Malarz ${ }^{17}$, Katarzyna Machnik ${ }^{18}$, Jolanta Goździk ${ }^{19}$

Mariusz Wysocki'

Tomasz Szczepański ${ }^{20}$

Department of Pediatric Hematology and Oncology, Collegium Medicum, Nicolaus Copernicus University Toruń, Bydgoszcz, Poland Department of Pediatric Oncology and Hematology, Institute of Pediatrics, Jagiellonian University Collegium Medicum, Kraków, Poland Deparment of Pediacic Oncology Hemalond Deparment of Pediatric Oncology, Hematology and Transplantology, University of Medica Sciences, Poznań, Poland Department of Pediatric Stem Cell Iransplantation, Hematology and Oncology Medical University, Wroctaw, Poland ${ }^{5}$ Department of Pediatric Oncology, Hematology epartment or Pedichicon and Diabetology, Medical University, Lodz, Poland DDepartment of Oncology, Children's Memorial Health Institute, Warszawa, Poland 'Department of Pediatric Hematology and Oncology, Medical University, Warszawa, Poland Department of Pediatric Oncology and

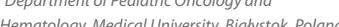
Hematology, Medicar University, Biatystok, Pola Department of Pediatrics, Hematology and Oncology, Medical University, Gdańsk, Poland ${ }^{10}$ Department of Pediatric Hematology, Oncology and Stem Cell Transplantation, Medical University, Lublin Poland

"Department of Oncology and Surgical Oncology for Children and Youth, Institute of Mother and Child, Warszawa, Poland

2Department of Pediatric Hemato-Oncology and Gastroenterology, Pomeranian Medical University, Szczecin Poland

${ }^{13}$ Department of Pediatric, Oncology and Immunology, Pomeranian Medical University, Szczecin, Poland

${ }^{4}$ Department of Pediatric Oncohematology, Rzeszów University, Rzeszów, Poland ${ }^{15}$ Division of Pediatric Hematology and Oncology, Children Hospitat Olsztyn Poland ${ }^{16}$ Division of Pediatric Hematology and Oncology, Children Hospital, Kielce, Poland "Division of Pediatric Oncology, Hematology and Chemotherapy, Department of Pediatric, Silesian Medical University, Katowice, Poland :s Division of Pediatric Hematology and Oncology Chision of Perictic Hew Chorzów Pediatric and Oncology Center, Chorzów Poland

${ }^{19}$ Department of Clinical Immunology and Transplantology, Jagiellonian University Medical College, University Children's Hospital in Kraków Kraków, Poland

Department of Pediatric Hematology and Oncology, Zabrze, Medical University of Silesia, Katowice, Poland

\footnotetext{
* Corresponding author: Jan Styczyński, Department of Pediatric Hematology and Oncology, Collegium Medicum, Nicolaus Copernicus University in Toruń, Skłodowskiej-Curie 9, 85-094 Bydgoszcz, Poland, phone: +4852 5854860, e-mail: jstyczynski@cm.umk.pl
} 
assess each center's strategy on testing parents staying with children in the hospital as well as medical staff (physicians and nurses) in the $\mathrm{PHO}$ ward, an additional questionnaire was conducted reporting the status as on July 1.

\section{Participating centers}

Children with oncological diseases in Poland are treated in 18 centers accredited by the Polish Society of Pediatric Oncology and Hematology. One center is split into two separate departments, and since both are located in the same building, they were calculated as one. In six centers, hematopoietic cell transplant (HCT) units are included.

\section{Patients at risk}

In Poland, approximately 1,100-1,200 children are diagnosed with malignant disease annually [8]. The total number of beds in Polish hemato-oncological and/or transplant pediatric centers is 725 , including 50 transplant units (single bed units only). Daily, an average number of hospitalized cancer patients, including children undergoing or after transplantation is 450, 1,100-1,200 monthly with an average of 100 newly diagnosed patients each month. The inaccuracy of estimation is below $10 \%$ (data not published, collected in July-August 2019 by the National Consultant in pediatric oncology and hematology).

\section{Testing for SARS-CoV-2}

Tests were based on molecular testing by real-time polymerase chain reaction (RT-PCR) of a specimen obtained from naso-pharyngeal swabs. Cases were analyzed in regional laboratories, designated and specified by the health authority.

\section{Definitions}

- Patient at risk: patients with a diagnosis of malignancy being on intensive chemotherapy (including periods off chemotherapy) or patients after hematopoietic cell transplantation at any phase being on immunosuppression or requiring visits to hospital.

- Clinical course of COVID-19 was determined according to Dong et al. [9] (Tab. I).

\section{Bioethical issues}

The study was performed in accordance with the medical principles of the Declaration of Helsinki and Good Clinical Practice guidelines. All participants of the survey are coauthors of this manuscript and gave their agreement for publication.

\section{Results}

\section{Epidemiological data}

With the announcing of the epidemic in Poland (March 23), the epidemiological analysis for incidence of SARS-CoV-2 positivity was performed in $\mathrm{PHO}$ centers among patients at risk, respectively,
Table I. Clinical course of COVID-19 [9]

\begin{tabular}{|c|c|}
\hline Clinical course & Description \\
\hline Asymptomatic & $\begin{array}{l}\text { Course without presence of any clinical symptoms and } \\
\text { signs. Chest imaging normal. Polymerase chain reaction } \\
\text { (PCR) test for SARS-CoV-2 positive }\end{array}$ \\
\hline Mild & $\begin{array}{l}\text { Symptoms of current acute upper respiratory tract } \\
\text { infection, usually including fever, fatigue, cough, } \\
\text { myalgia, sore throat, sneezing, and runny nose. Physical } \\
\text { examination: congestion of the pharynx, no auscultory } \\
\text { abnormalities. Sometimes no fever is seen. Possibly, only } \\
\text { gastro-intestinal tract symptoms may be present: nausea, } \\
\text { vomiting, diarrhea, abdominal pain }\end{array}$ \\
\hline Moderate & $\begin{array}{l}\text { Pneumonia, usually fever and dry cough, followed by } \\
\text { possibly productive cough, even with wheezing is seen. } \\
\text { No evident hypoxemia is present. Some cases may } \\
\text { exceptionally have no clinical signs and symptoms, } \\
\text { and only chest computed tomography (CT) shows lung } \\
\text { lesions }\end{array}$ \\
\hline Severe & $\begin{array}{l}\text { Early severe respiratory symptoms such as fever and } \\
\text { cough, sometimes accompanied by diarrhea or other } \\
\text { gastrointestinal symptoms. Usually in the second week, } \\
\text { the disease progresses to dyspnea and central cyanosis. } \\
\text { Hypoxia is present, with oxygen saturation below } 92 \%\end{array}$ \\
\hline Critical & $\begin{array}{l}\text { Patients progress to severe acute respiratory distress } \\
\text { syndrome (ARDS) and respiratory failure. Septic shock and } \\
\text { life-threatening organ dysfunction can occur, including } \\
\text { encephalopathy, acute kidney injury, heart failure, } \\
\text { myocardial injury, or coagulation dysfunction }\end{array}$ \\
\hline
\end{tabular}

for 2-week or 1-month periods (Tab. II). Only 14 patients were tested by March 23, and 72 patients by April 5 . The testing was quickly available in most of the centers, and by June 1 , already $15 / 18$ centers were able to test all patients admitted to the hospital (with some exceptions for 1-day treatments). During the analyzed period of 5 months, the overall number of estimated patients was 1,124 (ranging 1,073-1,233). During this period, the total number of patients diagnosed for SARS-CoV-2 positivity was eight (Tab. III). Thus, with the estimated total number of patients at risk at 1,5001,600 , the estimated incidence of SARS-CoV-2 positivity in Polish $\mathrm{PHO} / \mathrm{HCT}$ centers was $0.5 \%$. After exclusion of HCT patients (with one patient being infected), the estimated incidence of SARS-CoV-2 positivity is between 0.5 and $0.6 \%$. As of July 1 , parents staying in the hospital together with their children were regularly tested for the virus in 13 centers. Asymptomatic healthcare personnel were regularly tested for the virus in seven centers.

\section{Patients diagnosed positively for SARS-CoV-2}

Patient 1: 9-year-old girl, with relapsed acute lymphoblastic leukemia (ALL), after total body irradiation (TBI) and hematopoietic cell transplantation from matched unrelated donor (MUD-HCT), diagnosed on day +7 during neutropenia, had mild respiratory symptoms. She was treated with chloroquine, ribavirin, and azithromycin. After granulocyte recovery on day +14 , she recovered and was negative for SARS-CoV-2 twice after 7 and 8 days from diagnosis.

Patient 2: 5-year-old boy, with ALL, on chemotherapy (AIEOPBFM-2017, protocol II), hospitalized in the Department of Infectious Diseases with suspicion of encephalitis due to neurological symptoms (hemiparesis, facial nerve palsy, convulsions), and lung multiple nodules. Initially, tests for SARS-CoV-2 were negative twice, then positive (within 1 week from first symptoms). 
Table II. Estimated number of patients tested and diagnosed for SARS-CoV-2

\begin{tabular}{|l|l|l|l|}
\hline Date & $\begin{array}{c}\text { Estimated } \\
\text { number of } \\
\text { patients at risk }\end{array}$ & $\begin{array}{c}\text { Estimated number } \\
\text { of patients tested } \\
\text { by PCR }\end{array}$ & $\begin{array}{c}\text { Positive testing } \\
\text { (new) }\end{array}$ \\
\hline $23-03-2020$ & 1,073 & 14 & 0 \\
\hline $05-04-2020$ & $1,017\left(34^{*}\right)$ & 72 & 2 \\
\hline $19-04-2020$ & $1,056\left(47^{*}\right)$ & 292 & 0 \\
\hline $03-05-2020$ & $1,111\left(51^{*}\right)$ & 544 & 1 \\
\hline $17-05-2020$ & $1,137\left(55^{*}\right)$ & 834 & 2 \\
\hline $01-06-2020$ & $1,180\left(78^{*}\right)$ & 974 & 0 \\
\hline $01-07-2020$ & $1,199\left(69^{*}\right)$ & All patients in & 0 \\
\hline $01-08-2020$ & $1,233\left(56^{*}\right)$ & 944 & 3 \\
\hline
\end{tabular}

* - HCT patients at risk, included in overall number of patients

Cerebrospinal (CNS) fluid was normal. No respiratory insufficiency occurred and he finally recovered after 40 days; however, the last lung computed tomography (CT) performed 100 days from COVID-19 diagnosis revealed an increasing number of nodules along with persisting, but less pronounced fibrotic streaks, consolidation and discreet ground-glass opacities.

Patient 3: 5-year-old girl, with relapsed Wilms Tumor, immediately after cessation of therapy was asymptomatic, and did not require hospital treatment.

Patient 4: 12-year-old boy, with Down syndrome, treated for relapsed ALL, had mild clinical course, and did not require hospital treatment. Patient 5: 4.5-year-old boy, with Wilms Tumor, after preoperative chemotherapy and surgery, was asymptomatic, and did not require additional hospital treatment.

Patient 6: 4.5-year-old old boy, treated with chemotherapy for CNS tumor, was asymptomatic, and did not require hospital treatment.

Patient 7: 17-year-old, girl, treated for ALL on maintenance phase, with cough, olfactory, and taste disturbances was hospitalized in the Department of Infectious Diseases. She recovered after 14 days from the beginning of clinical symptoms.

Patient 8: 16-year-old, boy with osteosarcoma, during post-surgery chemotherapy, was hospitalized for fever and pneumonia with mild symptoms. He recovered after 10 days from clinical symptoms.

\section{Strategic management of pandemic in the PHO centers}

The strategic management of pandemic in the PHO/HCT centers was analyzed based on a questionnaire conducted on July 1 , among the Heads of the respective departments. All 18 centers (including HCT wards) filled in the questionnaire fully. Infection with SARS-CoV-2 occurred in hospitalized patients in one center, in outpatient patients in two centers, and in healthcare staff in one center. Hospitals implemented standard operating procedures (SOP) on prevention of infection as guidelines for personnel in all 18 centers, triage of patients in all 18 centers, and temperature measurements in all 18 centers. Additional preventive procedures were implemented in the PHO departments for patients and personnel. Phone triage was done in 14 centers, and testing for SARS-CoV-2 by PCR in 15 centers. Personnel rotation in various forms was performed in 13 centers and healthcare personnel testing for SARS-CoV-2 by PCR in 11 centers. The number of patients in PHO/HCT wards has decreased: the number of hospitalized patients decreased in 11 centers, with an estimated median value of $30 \%$ (ranging 15-50\%); the number of patients hospitalized on a 1-day basis decreased in 8 centers, with an estimated median value of $50 \%$ (ranging $20-70 \%$ ); and the number of out-patient patients decreased in 16 centers, with an estimated median value of $30 \%$ (ranging $20-100 \%$ ). Additionally, one center reported $30 \%$ decreased accessibility to laboratory diagnostics, and two centers a $15-20 \%$ decrease in accessibility to imaging diagnostics. No center reported any decrease in accessibility to medicines during this period.

\section{Discussion}

This nationwide study of SARS-CoV-2/COVID-19 status in PHO/ HCT centers showed relatively low estimated incidence of SARSCoV-2 positivity and high rate of centers performing testing for virus for all patients and their parents, when admitted to hospital (excluding 1-day hospitalization for chemotherapy, without overnight stay).

The estimated incidence of infection with SARS-CoV-2 ranged between 0.5 and $0.6 \%$ of all patients at risk in the $\mathrm{PHO} / \mathrm{HCT}$ centers. This rate is slightly lower in comparison to the largest COVID-19 database on PHO/HCT children run by the AIOEP/BFM consortium [10] (Ondrej Hrusak, personal communication, update open-online for participating centers), including over 6,700 patients. The incidence was in the range of $0.74-0.82 \%$, including Polish patients. This difference in incidence can reflect two important factors. First, lower overall incidence and number of infected individuals in the general Polish population, in comparison to Western European countries. Second, specific contact restrictions and set of anti-infective approaches implemented in Polish PHO centers, immediately after a rise in the rate of SARS-CoV-2 infections in Western countries (particularly Italy) in the initial phase [7]. Based on the EBMT [6] and Italian [7] experiences, the Polish Society of Pediatric Oncology and Hematology announced a national recommendation for the COVID-19 prevention and control in the pediatric units of hematology and oncology, hematopoietic cell transplantation units, and the respective outpatient clinics [11].

A total number of eight $\mathrm{PHO} / \mathrm{HCT}$ patients were infected with SARS-CoV-2, including one patient after HCT, infected during $\mathrm{TBI}$ conditioning regimen in another hospital. In all but one case the course of COVID-19 was asymptomatic or mild, and it was moderate in one case. None of them developed SARS or respiratory insufficiency, nor required treatment in the Intensive Care Unit, and no patient died due to SARS-CoV-2 infection. In the AIOEP/BFM consortium experience, the death rate was $4 \%$ (2/50 patients); both the deceased patients had co-infections with other viruses (influenza, parainfluenza, cytomegalovirus [CMV]).

As far as the practical aspects of preventive measures of infections with SARS-CoV-2 are concerned, the PHO/HCT wards and their hospitals implemented national regulations introduced by the government. In the majority of centers, additional testing of parents hospitalized with their children was also applied, and in half of the centers regular screening of healthcare personnel of $\mathrm{PHO} / \mathrm{HCT}$ wards took place. 
Table III. Clinical course in patients positive for SARS-CoV-2

\begin{tabular}{|c|c|c|c|c|c|c|c|c|}
\hline Pt & $\begin{array}{l}\text { Age } \\
\text { [yrs] }\end{array}$ & Sex & Severity of COVID-19 & $\begin{array}{l}\text { Days to } \\
\text { recovery }\end{array}$ & $\begin{array}{l}\text { Treatment for } \\
\text { COVID-19 }\end{array}$ & Comorbidities & $\begin{array}{l}\text { Type of } \\
\text { malignancy }\end{array}$ & $\begin{array}{l}\text { Oncological } \\
\text { treatment }\end{array}$ \\
\hline 1 & 9 & $\mathrm{~F}$ & Mild & 7 & $\begin{array}{l}\text { Chloroquine, } \\
\text { azithromycin, } \\
\text { ribavirin }\end{array}$ & No & Relapsed ALL & $\begin{array}{l}\text { After MUD-HCT } \\
\text { day+7 }\end{array}$ \\
\hline 2 & 5 & M & $\begin{array}{l}\text { Moderate (neurological } \\
\text { symptoms and pneumonia) }\end{array}$ & 18 & No & No & ALL & $\begin{array}{l}\text { Intensive } \\
\text { chemotherapy }\end{array}$ \\
\hline 3 & 5 & $\mathrm{~F}$ & Mild & 10 & No & No & $\begin{array}{l}\text { Relapsed Wilms } \\
\text { Tumor }\end{array}$ & $\begin{array}{l}\text { After } \\
\text { chemotherapy } \\
\text { cessation }\end{array}$ \\
\hline 4 & 12 & M & Asymptomatic & 5 & No & Down syndrome & Relapsed ALL & $\begin{array}{l}\text { Intensive } \\
\text { chemotherapy }\end{array}$ \\
\hline 5 & 4.5 & M & Asymptomatic & 21 & No & No & Wilms Tumor & $\begin{array}{l}\text { Intensive } \\
\text { chemotherapy } \\
\text { (after surgery) }\end{array}$ \\
\hline 6 & 4.5 & M & Mild & 14 & Azithromycin & No & CNS Tumor & Chemotherapy \\
\hline 7 & 17 & $\mathrm{~F}$ & Mild & 10 & Azithromycin & No & ALL & $\begin{array}{l}\text { Maintenance } \\
\text { chemotherapy }\end{array}$ \\
\hline 8 & 16 & M & Mild & 14 & Azithromycin & No & Osteosarcoma & $\begin{array}{l}\text { Post-surgery } \\
\text { chemotherapy }\end{array}$ \\
\hline
\end{tabular}

Pt - patient number; yrs - years; ALL - acute lymphoblastic leukemia; CNS - central nervous system; MUD-HCT - hematopoietic cell transplantation from matched unrelated donor

The majority of centers experienced a decrease in the number of patients admitted to the hospital, and almost all the centers in the outpatient setting. This was largely dependent on practices implemented by hospitals to limit personal contacts and the use of phone triage. This approach significantly contributed to the low incidence of SARSCoV-2 infections. Fortunately, no increase in mortality of patients with malignant diseases or undergoing hematopoietic cell transplantation was reported during this period. This observation undoubtedly underlines a very high level of medical service in the $\mathrm{PHO} / \mathrm{HCT}$ centers.

In conclusion, the estimated incidence of SARS-CoV-2 infection among $\mathrm{PHO} / \mathrm{HCT}$ patients is lower than in Western Europe; however, these patients cannot be regarded as a low-risk group. The low COVID-19 incidence should be interpreted as a result of strictly and continuously implemented detailed preventive measures in $\mathrm{PHO} /$ HCT wards and in hospitals.

\section{Acknowledgments}

The authors thank Prof Lidia Gil for preparing the questionnaire on the strategy of prevention of SARS-CoV-2 infections, and for the critical review of the manuscript.

\section{Authors' contributions}

JS - design of the study, writing manuscript. All authors - providing of data, analysis, critical revision, final approval.

\section{Conflict of interest}

All authors have nothing to disclose.

\section{Financial support}

None.

\section{Ethics}

The work described in this article has been carried out in accordance with The Code of Ethics of the World Medical Association (Declaration of Helsinki) for experiments involving humans; EU Directive 2010/63/ EU for animal experiments; uniform requirements for manuscripts submitted to biomedical journals.

\section{References}

[1] Cummings MJ, Baldwin MR, Abrams D, et al. Epidemiology, clinical course, and outcomes of critically ill adults with COVID-19 in New York City: a prospective cohort study. Lancet 2020;395:1763-70.

[2] Styczyński J. Hematology and transfusion medicine facing COVID-19: perspectives beyond the pandemic. Acta Haematol Pol 2020;51:58-9.

[3] Styczyński J. ABC of viral infections in hematology: focus on herpesviruses. Acta Haematol Pol 2019;50:159-66.

[4] Gil L, Kałwak K, Piekarska A, Góra-Tybor J, et al. Antifungal management in adults and children with hematological malignancies or undergoing hematopoietic cell transplantation: recommendations of Polish Society of Hematology and Blood Transfusion, Polish Society of Pediatric Oncology and Hematology, and Polish Adult Leukemia Study Group, 2020. Acta Haematol Pol 2020;51:60-72.

[5] Styczyński J. Infectious complications in children and adults with hematological malignancies. Acta Haematol Pol 2019;50:167-73.

[6] Ljungman P, Mikulska $M$, de la Camara $R$, et al. The challenge of COVID-19 and hematopoietic cell transplantation; EBMT recommendations for management of hematopoietic cell transplant 
recipients, their donors, and patients undergoing CAR T-cell therapy. Bone Marrow Transplant 2020;1-6 (online ahead of print).

[7] Balduzzi A, Brivio E, Rovelli A, et al. Lessons after the early management of the COVID-19 outbreak in a pediatric transplant and hemato-oncology center embedded within a COVID-19 dedicated hospital in Lombardia, Italy. Estote parati. Bone Marrow Transplant 2020;55:1900-5.

[8] Styczyński J, Balwierz W, Dembowska-Bagińska B, et al. Pediatric oncology and hematology in Poland. Pediatr Pol 2018;93:451-61.

[9] Dong Y, Mo X, Hu Y, et al. Epidemiology of COVID-19 among children in China. Pediatrics 2020;145:e20200702.
[10] Hrusak O, Kalina T, Wolf J, et al. Flash survey on severe acute respiratory syndrome coronavirus- 2 infections in paediatric patients on anticancer treatment. Eur J Cancer 2020;132:11-6.

[11] Styczyński J, Balwierz W, Wachowiak J, et al. The national recommendation for the COVID-19 prevention and control in pediatric units of hematology and oncology, hematopoietic cell transplantation units and respective outpatient clinics: guidelines of Polish Society of Pediatric Oncology and Hematology. Przegl Pediatr 2020;49:24-9. 